\title{
Aminoacyl-tRNA synthetases may have evolved from molten globular precursors
}

\author{
Zhijie Li $^{l}$, Charlie Carter ${ }^{l}$ \\ ${ }^{1}$ Department of Biochemistry and Biophysics, University of North Carolina, Chapel Hill, NC 27599-7260 \\ zhijie@ad.unc.edu
}

To represent an early stage in the evolution of contemporary aminoacyl-tRNA synthetases (aaRSs), the structurally invariant 120 130-residue Urzymes (Ur-: prefix for primitive, original) consisting of a minimal catalytic domain that has been re-designed and retains a considerable fraction of the catalytic activity of the native aaRSs. We previously described experimental evidence that the Urzymes exhibit $\sim 60 \%$ of the contemporary catalytic proficiencies and acylate tRNA far faster than the uncatalyzed rate of nonribosomal peptide bond formation from activated amino acids. Previous studies on the TrpRS Urzyme renatured from inclusion bodies suggested that it functions as a catalytically active molten globule ${ }^{[1]}$. This study focuses on LeuRS Urzyme, which is also purified from inclusion bodies. The NMR analysis suggests that LeuRS Urzyme is also a molten globule. As with the TrpRS Urzyme, its poorly-dispersed HSQC spectrum differs from those of well-folded proteins, indicating that it lacks extensive tertiary structure. Functional assays confirm that LeuRS Urzyme is catalytically active. As a molten globular catalyst, LeuRS Urzyme likely achieves complementarity to the transition state for leucine activation through a higher cost in entropy than that expected for a well-folded protein. Its catalysis therefore implies that even more favorable enthalpic changes must develop in the TS complex, by virtue of the added structural flexibility. Therefore, it is expected that some global conformational ordering of LeuRS Urzyme should be observed upon binding of a transition state analog ${ }^{[2]}$. We are exploring various potential inhibitors with this in mind.

\section{References}

[1] Sapienza, P. J., Li, L., Williams, T., Lee, A. L., \& Carter Jr, C. W. (2016). An ancestral tryptophanyl-tRNA synthetase precursor achieves high catalytic rate enhancement without ordered ground-state tertiary structures. ACS chemical biology, 11(6), 1661-1668.

[2] Vamvaca, K., Vögeli, B., Kast, P., Pervushin, K., \& Hilvert, D. (2004). An enzymatic molten globule: efficient coupling of folding and catalysis. Proceedings of the National Academy of Sciences, 101(35), 12860-12864. 